\section{LA CONSTRUCCIÓN DE \\ UN SOCIALISMO ARGENTINO \\ EN TORNO A ALFREDO PALACIOS ${ }^{1}$}

THE CONSTRUCTION OF AN ARGENTINEAN

SOCIALISM AROUND ALFREDO PALACIOS

CARLOS M. HERRERA ·

Director del Centre de Philosophie Juridique et Politique de l'Université de Cergy-Pontoise, Francia.

\section{Resumen}

Este trabajo se propone estudiar la tentativa de organizar un movimiento socialista integrando el componente nacional a su ideario. proyecto se materializó en torno a la figura de Alfredo L. Palacios, luego de su salida del PS. El nuevo programa podía poner en entredicho la cultura política del socialismo argentino, en un momento en que éste consolidaba su presencia en el sistema político. Sin embargo, la nueva identidad no logró cuajar en una organización duradera. Estas páginas exploran las razones.

\section{Registro bibliográfico}

HERRERA, CARLOS M. «La construcción de un socialismo argentino en torno a Alfredo Palacios», en: ESTUDIOS SOCIALES, revista universitaria semestral, año XXVIII, $n^{\circ}$ 55, Santa Fe, Argentina, Universidad Nacional del Litoral, julio-diciembre, 2018, pp. 91-120.

\footnotetext{
${ }^{1}$ Los intercambios con Patricia Ledesma y Paco Reyes enriquecieron este trabajo durante su elaboración.
}

\begin{abstract}
This essay aims to explore the attempt to organize a socialist movement by integrating the national component to its ideology. The project sought to materialize around the figure of Alfredo L. Palacios, after his expulsion of the party. The new program could put into question the political culture of the argentinian socialism, at a time when it was consolidated in the political system of the country. However, the new identity failed to materialize into a lasting organization. These pages explores the reasons.
\end{abstract}

\section{Descriptores · Describers}

Partido Socialista / Palacios / nacionalismo / cultura política / identidad

Socialist Party / Palacios / nationalism / political culture / identity

Recibido: 015/04/2018 Aprobado: 10/06/2018 
«Estaban en ese espacio ambiguo,

al mismo tiempo inmediato y remoto,

en el que lo familiar se transfigura

y empieza a parecerse a lo desconocido».

(J.J. Saer).

En algunos ensayos sobre Juan B. Justo que marcaron durablemente la investigación hace unas décadas subyacía implícita la pregunta: ¿por qué no hubo socialismo nacional en la Argentina? El interrogante se había actualizado tras la experiencia en el poder del peronismo y, en un contexto más general, por la relación histórica que se había cimentado entre socialismo y descolonización desde finales de los años I940, donde las revoluciones «contra el Capital» de las que hablaba Gramsci se habían generalizado.

En un ensayo ya clásico, José Aricó señalaba que «el problema de la nación, de su identidad, de sus incapacidades, de la vinculación entre propuesta nacional y propuesta socialista» no había sido asumido por el Socialismo, sobre todo en el marco de esa "autocrítica» que la crisis de i930 había abierto, justamente «en el momento mismo en que el socialismo mostraba una capacidad inédita de fundirse con la única clase verdaderamente nacional» (ARICó, I998: I47). Las causas remontaban para él a la hipótesis de Justo, no tanto por no haber sido consciente del carácter semi-colonial del país -éste era un rasgo que el dirigente socialista compartía como buena parte de la cultura argentina anterior a los años I930-, como por su confianza en la transparencia entre capitalismo y parlamentarismo, que guiaban su «desprecio» por las formas inorgánicas de incorporación de las masas argentinas a la política (ARICÓ, I998: II8 y I23) ${ }^{2}$. Por cierto, la reflexión de Arico tenía un estatuto interno particular, de "transición»: si el arrière-fond de su pensamiento estaba fuertemente enraizado en aquellas inquietudes nacidas en sus años sesenta, ya aparecían las vetas de un nuevo horizonte "social-demócrata» que emergía en su generación tras la derrota de los «socialismos nacionales realmente existentes» en nuestro país.

${ }^{2}$ Al mismo tiempo, Aricó elogiaba su visión de la conciencia de clase de los trabajadores, «con características nacionales propias que le permitieran ser a su vez una síntesis histórica de la realización nacional» (ARICÓ, 1998: 95). 
En verdad, la investigación historiográfica sobre el socialismo argentino que se ha venido desarrollando a partir de los ańos 2000 (CAMARERo y HERRERA, 2005), ha matizado la imagen de un Partido monolítico y homogéneo, poniendo en un lugar sus fallas internas, los quiebres. Pero esta característica no tocaba únicamente a sus vicisitudes «externas» como organización política: de algún modo se veía reflejada asimismo en su propia identidad. No que no contase el Partido Socialista con una identidad propia; ésta incluso se encarnaba, como pocas fuerzas de la tradición de la II Internacional, en la figura y las enseñanzas de un hombre, este Juan B. Justo que terminaba transformándose en los relatos en el «fundador» del Partido, con rasgos cuasi bíblicos. Sólo la fuerza de esta identidad explica por qué el PS pudo resistir a poderosos embates «externos», estatales incluso, que buscaron quebrarlo, como ocurriría bajo el peronismo (HERRERA, 20I6). De alguna manera, sólo una implosión interna podía acabar con él, cuando esa identidad política se encontraba quebrantada, al ser identificada, por una parte significativa de sus miembros, como un obstáculo a la relación entre el Partido y los trabajadores y, por ende, al socialismo. Pero no por nada aquel grupo que, tras la división de I958, logró reconstruir la vieja identidad de la manera más completa de cara al pasado, aun con componentes que ya no parecerían tan centrales en la política argentina (como el laicismo), fue el que pudo sobrevivir como fuerza política, lo que suponía también proyectarla hacia nuevos problemas (como la Guerra Fría, la evolución de la socialdemocracia, etc.).

Retomando aquella vieja inquietud que ilustrara Aricó después de tantos otros, nos proponemos explorar un componente de la identidad socialista argentina que fue objeto de arduos debates desde sus inicios, y de variadas formas: el nacional. En el sistema de representaciones que toda identidad política encarna, aquel fue siempre un elemento de tensión dentro del socialismo. Pero tensión no quería decir «ausencia», como lo pretendió cierta vulgata historiográfica, sino una urdiembre compleja, donde, tras una afirmación determinante de aquel principio frente a las tradiciones de izquierda del siglo XIX -aquella en favor de la acción política y por ende de la ciudadanía hecha por Justo ya en el Congreso «constituyente» de I896, al que se le opuso el fundamento «internacionalista»-, se confrontó sobre sus sentidos y su alcance en el programa político hasta la segunda mitad de los años I940.

Lo haremos desde un ángulo acotado: examinando el proyecto a través del cual Alfredo L. Palacios buscó darle al factor nacional el lugar central en la identidad socialista tras su exclusión de las filas partidarias. Tomar esta vía puede resultar 
apropiado por más de una razón, no sólo porque muestra que la «cuestión nacional» es antigua, sino, sobre todo, porque Palacios trató de expresarla muy tempranamente como alternativa a la identidad modelada por Justo, incluso bajo la forma de una nueva organización política: el Partido Socialista Argentino, activo entre I9I5 Y I922 (HERRERA, 2OI8).

De hecho, lo que distinguía a Palacios no era tanto su visión de la nacionalidad en sí, cuyos componentes están al menos en ciernes en el grupo dirigente del Ps, recibiendo incluso un impulso significativo tras el Centenario y la integración del Socialismo en el sistema político, sino el plan de ponerlo como eje de una concepción socialista. Buscaba, en otros términos, darle por primera vez una proyección política de izquierdas al nacionalismo, cuyo carácter superaría el papel de integrador de clases sociales y programas políticos ${ }^{3}$. Esta se traducía en una radicalización del reformismo en el plano de la acción, y una visión pluriclasista del programa económico y aún social. Por cierto, lo que entiende Palacios por "política» va a revelarse muy estrecho, como ejercicio parlamentario, lo que condicionará la actividad partidaria, centrada en la propaganda electoral.

En definitiva, el interés de concentrarse en esta experiencia (independientemente del dato que hasta ahora nunca había sido estudiada con detalle ${ }^{4}$ ), reside en el hecho de constituir la única, sin duda, que buscó recrear una fuerza política a partir de una identidad socialista nacional en las primeras décadas del siglo xx.

${ }^{3} \mathrm{Si}$ bien pueden hallarse similitudes en la operación de apropiarse del relato nacional que lleva a cabo en esos momentos el radicalismo, estos apuntan ante todo a la integración de ciertos sectores sociales (los trabajadores, las clases medias) de manera a legitimar su propia empresa en una totalidad dada, mientras que Palacios entiende darle, además, un contenido proyectual, aun en los detalles, como la relación que veremos más abajo con el programa agrario de Rivadavia, asumido como tal por su partido. ${ }^{4}$ El principal problema de fuentes está relacionado por la ausencia de una publicación propia extendida a lo largo de la experiencia. El periódico La Acción $(L A)$ cumplirá estas funciones en el primer año de existencia de la nueva fuerza, pero pronto se transformará en vocero de uno de los grupos internos, que culminan con una crisis y la desaparición del periódico en marzo de 1917. Se ha señalado (GARCíA COSTA, 1998) la existencia de otra revista ligada al partido, Nueva Era, dirigida por Juan F. Mantecón, que había iniciado su difusión en 1914, pero no tenemos registro de su edición más allá de 1916. 


\section{IDENTIDAD/CULTURA/TRADICIÓN SOCIALISTAS}

Aunque no la recubre enteramente, la noción de identidad está estrechamente ligada a la idea de cultura política, como dos caras de la misma moneda 5 . La identidad supone ya la aglutinación de un conjunto de características, mientras que la cultura encarna sus expresiones dinámicas, dejando entrever la pluralidad más fácilmente. De lo que es, ante todo, un conjunto de prácticas, la identidad aparece como el hilo que permite articularlas como un todo. Se trata de una relación dinámica, en ningún momento unidireccional, porque las identidades operan a su vez sobre la cultura. Y si se quiere conservar la metáfora numismática, el canto entre ambas dimensiones lo constituye su historia, asumida bajo la forma de la tradición. Por cierto, una identidad tampoco se identifica absoluta y exclusivamente con los contornos de una corriente política, o en todo caso, suelen ser concéntricas y englobar identidades más vastas. Así, el laicismo pudo ser un componente del liberalismo, del socialismo y más tarde del comunismo.

Las identidades no suelen tener un origen preciso, se nos presentan más bien como sucesivas sedimentaciones que aparecen «estructuradas» a partir de un determinado momento, aquel que les permite desplegarse hacia afuera en un conjunto de sentidos específicos, que se proyectan incluso a asuntos que superan la cuestión inicial -así, la identidad «obrerista» del socialismo permitía dar respuesta al problema de las elecciones, de los símbolos patrios, de la organización interna, etc.-. Pero aun en aquellas fuerzas que presentaban un programa más sistemático y explícito, como el ps, las identidades se integran con culturas lo suficientemente porosas para convivir dentro de un mismo partido.

La identidad del Socialismo argentino estaba compuesta por múltiples capas, no siempre homogéneas; o en todo caso, su expresión dominante convivió, y combatió, con otras modalidades concurrentes, que nunca lograron trastocarla. Se puede avanzar como hipótesis que la clave del éxito de la identidad «justista» fue lograr nutrirse hábilmente de otros componentes para realizar una síntesis integral, que los ordenaba según una lógica interna muy atenta siempre a la evolución de la propia fuerza.

\footnotetext{
${ }^{5}$ Estas reflexiones se inscriben en una discusión historiográfica francesa, donde las culturas políticas aparecen como las forma(s) a través de las cuales un partido se dota de una identidad distintiva (SIRINELLI, 1998; BERNSTEIN, 1998 y 1999).
} 
Toda identidad política, dijimos, es un catalizador de múltiples componentes. Se trataba de un movimiento -en sentido de desplazamientos- que, tras nutrirse de numerosas culturas en su formación, las utilizaba luego en las fronteras de demarcación, de manera a proteger su núcleo central, y, al mismo tiempo, poder entrar en contacto con otras identidades políticas que desbordaban las filas partidarias. Ya en nuestro tema, el artículo de Justo «Por qué no me gusta escribir para una hoja que se dice israelita» (1923) es paradigmático de esa ondulación, de esa movilidad: al mismo tiempo que se reivindicaban los sentimientos nacionales en sentido propio, se afirmaba que «nuestro más acentuado carácter nacional es nuestro internacionalismo» ${ }^{6}$. Ya en I898, aunque condicionado tal vez por su auditorio -una conferencia en el Ateneo Nacional-, Justo había afirmado las coordenadas de su posición «argentina». No dudará, poco después, en presentar al socialismo como «el buen nacionalismo», por sus efectos en materia de ciudadanía, de independencia política y de educación de las masas, que hacen surgir «lo bueno y vital de la nacionalidad», incluso en el plano del poder militar.

Si la identidad más antigua de los socialistas fue la "obrerista», que ponía también en órbita el componente internacionalista, la «nacional» aparecía como más ardua, no sólo por su contenido sino por su función, en algún lugar metaconceptual, lo que facilitaba su función como tal. Este carácter más abstracto que la obrerista le permitió conjugarse con variadas disidencias, tanto las motorizadas por sus alas «derechas», como fue el caso del Partido Socialista Independiente en los años $1920^{7}$, como, aunque de manera más ocasional, por las izquierdas, como lo expresó, en la década del '30, el Partido Socialista Obrero, o en los años 1950, Acción Socialista. Como la identidad «obrera», también podía impulsar e integrar otros componentes específicos, como el «popular», o el «latinoamericano».

Por cierto, dentro del ps se asociaba a ciertas figuras públicas con la promoción de algunas de estas identidades, aunque esto no obstaba a que esos hombres pudiesen defender posiciones diversas y cambiantes de cara al debate interno. Así, la promoción de una identidad nacional pudo encarnarse en otros hombres que

\footnotetext{
${ }^{6}$ Allí sostenía, con ecos que tal vez hoy se considerasen antisemitas, que aquellos que se reivindicaban como judíos «lastiman mis sentimientos nacionales», por su rechazo a asimilarse, abandonando su identidad étnica, religiosa e incluso nacional

7 De hecho, su futuro líder, Antonio de Tomaso se contaba entre quienes promovían un nacionalismo socialista ya antes de la década de 1910 (REYES, 2018).
} 
Palacios, como de Tomaso o, con otros tintes, Mario Bravo, entre los dirigentes más antiguos, y posteriormente encontrará otros representantes «menores» como Julio V. González.

No buscamos aquí reconstruir las estaciones y el ideario de la identidad nacional del socialismo argentino, que supondría periodizar mejor los contextos históricos donde se daba, sino concentrarnos en una experiencia partidaria efímera pero que fue la primera que buscó encarnarla. Esta se desarrolló tras la separación de Palacios del ps -involucrado en un nuevo «lance de honor» iniciado en un incidente parlamentario con un legislador radical-, decidida en junio I9I5 por el Comité Ejecutivo, y ratificada, ante la apelación del diputado, en julio de aquel ańo, por el II Congreso extraordinario, que no hizo lugar a reconsideración alguna. A consecuencia de ello, Palacios renunció a la banca que ocupaba en el Congreso desde 1912. Paralelamente, pocas semanas más tarde, surgía un Partido Socialista Argentino, impulsado por un conjunto de delegados disidentes que habían defendido durante las deliberaciones de aquel Congreso de la Verdi la demanda de Palacios.

En verdad, ya antes de su adhesión al Partido, en 190I, Palacios buscó afirmar una visión propia del socialismo, que en su momento ahondará en la idea cristiana, o al menos en una cierta distancia con el marxismo. Además, en otro plano, trataba siempre de concitar apoyos a su persona en otros grupos organizados, cuya primera expresión serán los "Círculos de obreros liberales», un espacio reformista -una «nebulosa»-propia a ese tiempo, y que tenían como zócalo común la preocupación por el mejoramiento de las clases trabajadoras, o más aún la protección de los débiles en general (mujeres, niños), combatiendo las organizaciones prohijadas por la Iglesia Católica en esos momentos. Su inesperada elección a diputado, en 1904, se benefició de hecho de apoyos más dilatados -en particular de las fuerzas liberales de Emilio Mitre-, de los que le brindaban de por sí una circunscripción obrera como la $4^{\circ}$ sección (TORRE, I976).

Su práctica «exitosa» como legislador -como lo atestaba la sanción de normas como la ley de descanso dominical (1904) o la reglamentación del trabajo de las mujeres y los niños (1907)-, sobredeterminó algunos de los elementos de la identidad socialista, profundizando la centralidad política del Parlamento dentro del proyecto reformista. Se pueden ver las marcas de esta experiencia cuando en Teoría y práctica de la historia, fechada algunos años antes que la Ley Sáenz Peña, Justo escribía: 
«Al extenderse y normalizarse la acción política obrera, creará esta diputados de profesión, hombres hechos por el estudio y la experiencia para la acción parlamentaria, en la cual alcanzarán un grado de eficiencia bien superior la de los representantes aficionados y de ocasión que elige la burguesía [...] Y esta misma subordinación de su palabra en el parlamento a las ideas de sus representados, les dará más motivos y libertad para exponer opiniones propias, y rebatir el error ante sus electores en la conferencia y en los debates internos del partido» (JUSTO, I909: 429-430).

Justo potenciaba un punto de vista que estaba en discusión aun en otros partidos de la II Internacional ${ }^{8}$, siguiendo en esto las posiciones de Jean Jaurès, con cuyo «juridicismo», por otra parte, tenía mayores diferencias que Palacios (HERrERA, 20I5).

\section{EL NACIMIENTO DE UN NUEVO SOCIALISMO}

Ya en las vicisitudes que llevan a la salida de Palacios del Ps, con gran repercusión en la prensa nacional, aparecen algunos elementos que nos indican la dirección que tomará su proyecto político a mediados de 1915. Así, al responder a los discursos de un banquete que organizan los cronistas parlamentarios en su homenaje, y que tomaba rápidamente un cariz anti-socialista ${ }^{9}$, Palacios fijaba dos coordenadas teóricas de manera explícita. La primera es que coexiste con la lucha de clases una "cooperación de clases», que la atenuaba. Tomarla en cuenta alejaba de los fanatismos y los sectarismos, porque no podía sostenerse una doctrina en contra de los hechos. Justamente, había que adaptar las actuaciones a lo que llamaba «las condiciones del país», una vez más sin «intransigencias», lo que le permitía desplegar su segundo eje: la patria no era incompatible con el internacionalismo, reivindicando una nueva nacionalidad, que era producto del cruzamiento entre los viejos criollos y los inmigrantes.

El Partido Socialista Argentino (PS-A) nacía en un congreso de «agrupaciones socialistas disidentes» reunido el 4 y 5 de septiembre de 1915 por algunos de los

\footnotetext{
${ }^{8}$ Para Justo, la importancia de la ley era mayor en los países de inmigración reciente (JUSTO, 1909: 437).

9 Uno de los oradores, Ricardo Rojas, tras atacar violentamente a sus principales dirigentes, decía explicarse por qué habían abandonado el PS artistas como Lugones y Payró, científicos como Ingenieros y Arraga o quienes no renunciaban a su raza, como Ugarte.
} 
disconformes del Congreso de julio, en apariencia, de manera independiente a la voluntad de Palacios. Este, en un reportaje tras su separación del Ps, aclaraba que, aunque no había pretendido organizar una fuerza alternativa, había una serie de hombres que se habían alejado del socialismo "desde tiempo atrás» y que podían coincidir con «muchos elementos independientes que aceptan en todas sus partes las reformas de índole económica y política, pero sin darle mayor importancia al programa máximo». En ese sentido, se declaraba dispuesto a formar parte de un «partido socialista nacional» que, «sin hacer alarde de nacionalismo, sin explotarlo como bandera, tampoco tenga reparo en aceptarlo» ${ }^{10}$. En todo caso, Palacios no participaba en las deliberaciones de aquella asamblea; en cambio, una foto muestra a Alfredo J. Torcelli y Martín Casaretto presidiéndolas bajo la consigna «Igualdad, libertad, fraternidad» desde un escenario, donde se reconoce al fondo un retrato de Sarmiento ${ }^{11}$. Al finalizar sus debates con la adopción de un nuevo programa, se abría un breve juego de acercamiento entre la nueva organización y el antiguo diputado, del que incluso la gran prensa se hará eco, hasta que por carta del I6 de septiembre Palacios solicitaba su afiliación al Partido ${ }^{12}$. No se trataba completamente de una actitud fingida: había sin duda diferencias entre esos disidentes, que ostentaban, como Casaretto, posiciones de izquierda, y el ex diputado, que abogaba por una actitud más amplia y nacional. El Manifiesto inicial da las coordenadas del nuevo Socialismo, donde el reformismo se teñía con una sutil reivindicación de la solidaridad como horizonte:

«El país necesita más que nunca completar su emancipación para orientarse hacia sus futuros destinos. Cimentemos una democracia sin yugos; proyectemos al porvenir nuestras ocultas energías y afiancemos la solidaridad social sobre los sentimientos más nobles de la estirpe» (PALACIOS, I9I6: 424).

El viejo socialismo, en cambio, caía entre aquellas agrupaciones que mostraban «desconocimiento de las modalidades nativas». Pero también se subrayaba entre las razones que actualizaban el nuevo ideario la presencia de la Guerra mundial, que «obliga a los pueblos americanos a reflexionar sobre sus propios destinos». El

10 «Con el Dr. Palacios», El Diario, 12/7/1915.

11 «Socialistas disidentes», El Diario, 4/9/1915.

12 «Adhesión del Dr. Palacios», La Razón, 16/9/15. 
acta fundacional reivindicaba el pacifismo de dichos pueblos, que aparecía como el principal rasgo de su identidad en el contexto de la conflagración mundial. Aunque reivindicándose tributarios del pensamiento europeo, la Guerra -calificada como «desastre»- llevaba a reconocer más claramente "un destino histórico distinto», que llevaría a «emanciparnos del tutelaje para crear formas de vida superiores y armónicas».

Siguiendo la vía de la Independencia, se reivindicaba «la autonomía de nuestro pensamiento», para combatir, «con métodos propios», las desigualdades, en una perspectiva que mezclaba el constitucionalismo norteamericano y los descubrimientos de Ameghino, para resaltar el valor de «medios que nos sean exclusivos» de transformación, en todo caso, sin tomar como dogmas los modelos del socialismo europeo. Asimismo, y contra el determinismo, se asumía el colectivismo como un «objetivo" y no como una "finalidad del progreso humano", lo que habilitaba más claramente la acción política de tipo reformista. En efecto, un criterio práctico era puesto en el centro del ideario. En síntesis, se trataba de ofrecer una ruta «más amplia» a los trabajadores para la defensa de sus derechos, que pasaba por considerar al socialismo "desde nuestro punto de vista americano».

A su vez, el nuevo Partido condenaba al personalismo, que surgía como rasgo central de la historia política del Estado argentino, y que rebajaba al pueblo a un rol pasivo, aunque el documento se mostraba dispuesto a encontrar en esos procesos «las fuerzas directrices de nuestra embrionaria nacionalidad». Aunque no dejaba de reivindicar los cambios que produjo en la política argentina la llegada del Socialismo. Sus triunfos electorales capitalinos no sólo probaban la eficacia de sus métodos, también que su programa concitaba un apoyo más amplio que los trabajadores. Paradójicamente, era en esos momentos que el ps extravió su dirección: al dogmatismo y al sectarismo que había acendrado en él, «desencarnado de la tierra donde pretendía arraigar», seguían incluso prácticas de la política criolla.

Ante ello, el PS-A perseguiría el ideal socialista «dentro de las modalidades inherentes a nuestra democracia», lo que parecía anunciar una orientación reformista que demandaría reinterpretar un conjunto de conceptos (como lucha de clases, política, etc.) (HERrera, 20I8). Presentándose pues como una «fuerza que pretende ser genuinamente nativa y espontánea», ajustaría, en el plano táctico, «su propaganda y su acción a las modalidades del ambiente», lo que significaba «enaltecer los sentimientos y las virtudes primarias que trasunten la salud espiritual de la raza», y evitar el «dogmatismo sobrecargado de erudición pedantesca», promoviendo, en cambio, «la verdad escueta en lenguaje simple o regional». También renunciaría a 
predicar una moral trascendente, puesto la nueva fuerza se asumía como una parte de la opinión nacional, lo que se traducía, en el plano interno, en una disciplina de otra naturaleza que la practicada por imposiciones. En ese sentido, se trataba de un partido de individuos, e incluso de individualidades, antes que de familias o iglesias.

Pero la nacionalidad, cabe subrayarse, no se presentaba ya como un todo integrado, sino que surgiría de la convergencia entre argentinos y extranjeros. En esa perspectiva, el nuevo partido se proponía afianzar la solidaridad social «sobre los sentimientos más nobles de la estirpe». Por cierto, se juzgaba que el país presentaba hoy «todas las condiciones de la sociedad capitalista europea». La conexión con la historia nacional surgía de la necesidad de «completar» la emancipación del país, percibiendo la revolución no como una ruptura, sino como una evolución, visión que ya se afirmaba en el socialismo justista, pero que tendía a absolutizarse aquí. En efecto, la Declaración de Principios colocaba en la enfiteusis rivadaviana como un momento central de ese proceso histórico, al establecer el principio de la tierra como propiedad colectiva e inalienable.

¿En qué rompía el Programa Mínimo del nuevo socialismo con el del antiguo ps? Lo distinguía ante todo su mayor extensión y la especialización en ciertos puntos: dividido en cuatro partes, cada una de ellas con una problemática definida, marcaba muchas veces una actualización necesaria con respecto a la evolución del país. El primer punto cubría los aspectos económicos, empezando por el sistema fiscal, pidiendo la tradicional abolición de los impuestos que encarecían los consumos del pueblo, pero también el fin de las patentes que gravaban a las profesiones útiles, así como la instauración del impuesto progresivo a las sucesiones. Los mayores detalles aquí tocaban a la producción agropecuaria: impuesto general y progresivo sobre la renta, en particular del suelo, una genérica supresión de los impuestos que grababan la producción agrícola y ganadera, y la eliminación de la contribución directa para la pequeńa propiedad rural ocupada por sus dueños, en propuestas que recordaban las ideas del georgismo, en boga en esos momentos. Al mismo tiempo se retomaba una vez más la vieja prédica en favor de la estabilidad de la moneda.

La segunda parte era la más extensa e ilustraba el tropismo jurídico del partido. Se reclamaban una serie de reformas legales que tocaban en primer término a la legislación del trabajo, con consignas tradicionales como salario mínimo, limitación de la jornada de trabajo, semana inglesa, etc., y la emergencia de propuestas novedosas como la inspección de la fábrica confiada a los obreros designados por los gremios, y, de manera general, una particular atención a las mujeres. Éstas se 
extendían al seguro obrero (detallando las modalidades de un seguro obligatorio, la admisión del riesgo profesional, la fijación de pensiones, siempre con la garantía del Estado), a la higiene y seguridad del trabajo, al trabajo del campo (incluyendo el pago de las mejoras para los arrendatarios pero sobre todo la expropiación paulatina de $25 \%$ de cada latifundio para ser entregado en enfiteusis al modo rivadaviano) y finalmente a la inmigración, para la que se pedía el fin de todo fomento artificial, una preocupación constante del viejo $\mathrm{PS}^{13}$. Siempre en la veta jurídica, la tercera parte era dedicada exclusivamente a las reformas de carácter constitucional, haciendo más explícitas viejas propuestas del socialismo, como el sistema proporcional, la supresión de la organización federal (con el fin de las gobernaciones y legislaciones provinciales así como el senado), el establecimiento del sistema parlamentario de gobierno, la separación de la Iglesia del Estado y toda una serie de medidas anticlericales, y la nacionalidad para los extranjeros tras 2 años de residencia.

El último apartado, proponía una serie de reformas nuevamente separadas en capítulos: electoral, incluyéndose el sufragio universal sin exclusión de sexos o el referéndum de iniciativa popular, municipal -reclamándose la tradicional autonomía, así como la municipalización de los servicios públicos-, de los códigos civil y comercial -previendo la igualdad civil de ambos sexos, la igualdad entre los hijos, el divorcio absoluto y la modificación de la ley de quiebras reconociendo el privilegio general de los salarios y sueldos-, la del código penal -con la abolición de la pena de muerte y la publicidad del sumario-, la reforma militar -con la abolición del ejército permanente reemplazado por la milicia ciudadana-, y la de la instrucción pública, donde, desde la nacionalización de la enseñanza a la defensa de la escuela laica, encontramos tópicos habituales de la corriente.

Casi tan importante como esos documentos oficiales aprobados en el congreso partidario era la carta de adhesión de Palacios, que tomaba a veces la forma de la glosa de sus proposiciones, asegurando encontrar en la Declaración de principios las ideas que defendiera en el ps "con escaso éxito» ${ }^{14}$. Allí subrayaba la intención de conciliar «los altos ideales del socialismo» con «las modalidades características de nuestro ambiente», o como decía más explícitamente, «respetando el sentimiento nativo». Parecía incluso imaginar el rol del partido como un «comité que vele por

${ }^{13}$ Adolfo Dickmann la calificaba en esos momentos de «obstáculo real para el progreso político de la República», retardando también el desarrollo del movimiento obrero (DICKMANN, 1916: 90).

14 «Declaración de principios», LA, 10/11/1915. 
el cumplimiento de las leyes obreras, obtenidas por mí, del Congreso». Recalcaba en el documento la vinculación entre la doctrina socialista y «los organizadores de nuestra nacionalidad», viendo en el socialismo la «ampliación inteligente de lo que concibieron esos grandes varones», en particular Rivadavia que prefiguraba para Palacios las ideas colectivistas. En ese sentido, historiaba la acción del patricio desde I8I2, que mostraban que no las concebía de manera platónica. Y contra quienes habían interpretado esa enfiteusis como romana o feudal, Palacios insistía en que la propiedad tenía base colectiva, estableciendo la renta en favor de la sociedad, que asociaba al sistema puesto en práctica por Nueva Zelanda. Para Rivadavia, la renta del suelo podía reemplazar todos los impuestos e incluso la aduana. La misma preocupación social surgía de su preferencia por el impuesto a las sucesiones. En ese sentido, Palacios no dudaba en considerarlo «el padre del socialismo argentino». De esa tradición «honrosa», que "contiene el germen de la democracia argentina», pero que es ajena a todo "nacionalismo estrecho", Palacios rescataba, como venía haciendo su antiguo partido desde el Centenario, a Echeverría y a Alberdi, que habían visto la importancia del factor económico en la formación de la nacionalidad.

Aun celebrando en Marx al fundador del socialismo científico, Palacios reivindicaba a Eduard Bernstein en la certeza que la idea que el proletariado no tenía patria había quedada superada a medida que la influencia de la socialdemocracia llevado al obrero a ser «ciudadano cooperador de los bienes de la nación». Pero como siempre en su ideario, era Jaurès quien le servía para mostrar que internacionalismo y patriotismo no sólo no eran términos antagónicos sino que la idea de patria se revelaba indispensable para la concepción socialista, ya que la unidad nacional era la condición misma de la unidad de producción y propiedad, constituyendo así los puntos de apoyo y de partida para el socialismo. Aquí las tareas del socialismo parecían interpretarse en clave revisionista, como «elevar el nivel de vida del obrero, dignificar su trabajo, darle garantías y seguridades materiales, reconociendo el derecho a la vida y proclamando el principio de solidaridad social». En todo caso, Palacios llamaba a aceptar los pequeños pasos, sin extasiarse por los fines lejanos, definiendo la reforma como «la revolución sin el bluff», la «revolución en camino».

E iniciando la disputa por la identidad con el viejo ps, terminaba llamando a la aceptación de todas las formas psicológicas de la personalidad, que nunca podían poner en peligro la unidad del partido ${ }^{15}$.

\footnotetext{
${ }^{15}$ Esta había sido la línea de defensa que había utilizado contra su separación.
} 


\section{III. ¿QUÉ IDENTIDAD PARA UN SOCIALISMO NACIONAL?}

Las páginas del órgano partidario, La Acción ( $L A)$, permitían observar la emergencia del nuevo proyecto pero mostrarán también sus tensiones internas. El periódico era dirigido por uno de los hombres experimentados que rodeaban a Palacios desde sus inicios en la actuación pública, Alfredo Torcelli. Más allá de su calidad de vocero oficial, pronto $L A$ aparecerá como representante del sector más cercano a las viejas tradiciones socialistas, aunque sus páginas estaban abiertas a colaboraciones más amplias.

En diapasón con su Declaración de Principios, el periódico iniciaba su papel de tribuna de la nueva doctrina bajo la admonición de "un regreso a Rivadavia», cuya imagen ilustraba la portada del primer número ${ }^{16}$, y al que calificaba de "genial factor de civilización», siendo incluso parangonado alguna vez con Marx. Si Rivadavia había denunciado en la apropiación de la tierra la fuente de las desigualdades humanas, la importancia de su enfiteusis, como se subrayaba desde el primer número, era nacional: concebía la tierra como patrimonio común de todos los argentinos, dando como base a la nueva nación su propiedad colectiva, y con ello, la democracia ( $\left.\mathrm{n}^{\circ} \mathrm{I}\right)$. Más tarde, Carlos G. Antola consagrará tres artículos a analizar «el colectivismo agrario de Rivadavia». Esta reivindicación de

${ }^{16}$ Cada número, hasta fines de 1916, contenía un retrato en ovalo en la portada, donde se entrecruzan un panteón nacional -Echeverría (presentado como un precursor del socialismo en la Argentina), Alberdi, Sarmiento, la tríada que junto a Rivadavia formarían las bases del socialismo argentino, y más tarde Moreno o Castelli-, la tradición socialista -el gran referente internacional del PS-A Jaurès, y August Bebel, Liebknecht, Lassalle, Laffargue pero también Engels, Becker, Carlo Cafiero, Edouard Vaillant, Andrea Costa e incluso nombres menos conocidos como el diputado socialista italiano Pedro Chiesa, o más antiguos, como Saint Simon u Owen- y H. George. Más tarde habrá un latinoamericano en la persona de José Martí. Además, completan la galería sabios al uso (como Copérnico, Galileo, Leonardo, Franklin, Mirabeau, Darwin, A. Humboldt, Ameghino, Pasteur, Charcot, Cesare Cantú, José de Echegaray), escritores cercanos a la cultura socialista (como La Fontaine, Voltaire, E. de Amicis, Tolstoi, Zola, Victor Hugo, Shakespeare, Ibsen, Tasso, Zorrila). Se subrayará también la elaboración de una cultura científica social, que aparece en la sección «Los grandes sociólogos», bajo la pluma de Miguel Aquino, por donde desfilan perfiles de Adam Smith, Jean-Baptiste Say, Robert Owen, Charles Fourier, David Ricardo, Saint Simon, Bastiat, Proudhon, Rodbertus, John Stuart Mill, Marx (bastante tardíamente, en el $n^{\circ} 13$, aunque se repite en el $n^{\circ} 19$ ), Lassalle, Bakounine, Henry George... Esta galería, donde conviven liberales, socialistas de diversos períodos, especialistas de política social, da cuenta de una pluralidad ideológica, y sobre todo, una demarcación más amplia de lo que es socialismo. Más adelante (partir del $\left.n^{\circ} 24\right)$, se reproducen viñetas sobre temas claves del Partido, como la oposición al militarismo. 
la enfiteusis rivadaviana llevaba a incluirla en las consignas electorales de 1916, ya que lo esencial del programa del PS-A se construía en torno a la cuestión de la propiedad de la tierra. Y siguiendo la línea que de algún modo Justo había hecho explícita en el Congreso de Junín, el Socialismo argentino reivindicaba la pequeña propiedad para democratizar la estructura económica del país ( $\left.\mathrm{n}^{\circ} \mathrm{I} 4\right)$, con consignas «aumentando el número de propietarios, disminuyen el número de los proletarios». Aparte, el tipo de cultivo que permitía guiaba la economía hacia el bienestar y no la opulencia. Sin contar, como se podía leer también, que la pequeña propiedad favorecía el orden.

Esto no implicaba renunciar a la difusión de las doctrinas del socialismo científico, pero debía ser actualizado por las verdades «experimentalmente adquiridas en el campo de la sociología». En su Manifiesto del I ${ }^{\circ}$ de mayo de 1916 se subrayaba que el socialismo, tanto como doctrina como ideal, «no es ni puede ser un monopolio exclusivo de una clase o de un partido», pudiéndose encarnar en todos los espíritus. No por casualidad ponía a la «legislación social» como la primera significación del $\mathrm{I}^{\circ}$ de mayo $\left(\mathrm{n}^{\circ} 24\right)$. En su discurso en esa fecha, Palacios estimaba que se debía hacer "socialismo nacionalista», al menos hasta que el internacionalismo tuviese la fuerza de realizar sus propósitos, cosa que la Guerra mundial ponía en entredicho. Se trataba con todo simplemente de unir a los obreros de la República para obtener dentro de ella los beneficios a los que tenían derecho.

Así como no anula al individuo, tampoco el socialismo excluía a las patrias, amparándose una vez más a la autoridad de Jaurès. Al contrario, exigía, según el PS-A «que se desarrollen en cada una de las patrias nacionalidades vigorosas y sanas», capaces de ser los pilares del futuro. Ya en su Congreso constituyente, el Partido había adelantado su voluntad de afiliarse a la Internacional obrera de Bruselas, una vez que la situación producida por la Guerra volviera a su cauce. El internacionalismo para el PS-A comenzaba con un afianzamiento de la unidad nacional, lo que significaba "conciliar los principios del socialismo integral con el sentimiento nativo y las modalidades de nuestro pueblo» ( $\left.n^{\circ} 15\right)$. En efecto, la finalidad internacionalista y universalista no anulaba el «buen nacionalismo», elevando a nativos y extranjeros en el pensamiento y extendiendo la acción hacia América (sin adjetivos, aunque parecía ser la hispana). «Conquistemos la redención 
para los pueblos, los pueblos para las patrias, y las patrias para la Humanidad» era una consigna repetida a lo largo de su actuación pública ${ }^{17}$.

Raras veces ese nacionalismo alcanzaba una traducción más precisa en el plano económico. Una nota sin firma denunciaba que el país era una "factoría inglesa» $\left(\mathrm{n}^{\circ} 25\right)$, ya que «las instituciones, las autoridades y el pueblo nacional y extranjero carecen de todo amparo y de cualquier derecho», ante el capital británico, como lo mostraban las huelgas ferroviarias. Más adelante, un editorial afirmaba que Argentina podía ser un país industrial, dada la calidad y cantidad de las materias primas, lo que permitiría incluso la exportación de productos. Pero este estadio se debía alcanzarse gracias a otros instrumentos que los subsidios protectores, incluso la instrucción cívica $\left(\mathrm{n}^{\circ} 37\right)$. De algún modo, estas caracterizaciones no rompían con las tradicionales propuestas del viejo socialismo en el plano económico. Así, en materia fiscal, los Argentinos rechazaban el proteccionismo que creaba industrias nacionales artificiales. Al mismo tiempo que se exigía la supresión de los impuestos al consumo del pueblo, se promovían dos tipos de impuestos, el directo y progresivo a la renta en géneros y a la renta de la tierra, y el impuesto progresivo a la herencia ( $\mathrm{n}^{\circ} \mathrm{I} 8$ ), de manera a evitar la demagogia de los caudillos, pagada por presupuestos excesivos.

Casi tanto como un partido nacionalista, el Ps-A se perfilaba asimismo como un partido de juristas. Incluso, se traducían las propuestas de su Programa mínimo en favor del mejoramiento económico y social de los trabajadores como la aspiración de "garantizar el derecho a la existencia», para aquellos que sufren la «irritante desigualdad económica» ( $\mathrm{n}^{\circ}$ I3). La precisión jurídica con que habían sido redactadas las propuestas de su Programa, tanto en materia de legislación del trabajo, desglosado del Seguro obrero, de las normas de Higiene y seguridad del trabajo, y del trabajo del campo, como en materia de derecho constitucional, donde se incluía el régimen parlamentario de gobierno, la supresión del Senado, sin hablar del detalle de las reformas promovidas en los diferentes códigos daban cuenta del origen de sus dirigentes. En momentos de la campaña electoral de i9i6, el PS-A no dudaba en subrayar que el autor de las 16 leyes obreras era un afiliado suyo.

\footnotetext{
${ }^{17}$ Sin duda la doctrina de Jaurès, tan contraria a la guerra -se publicará el último discurso antes de su asesinato- como a la negación de la nación como instancia política necesaria a la construcción del socialismo, podía ser durante cierto tiempo un punto de encuentro entre las diversas sensibilidades del nuevo Partido.
} 
Por cierto, el periódico dedicaba largas notas sobre la legislación social, que se convierte casi en su materia principal, aunque la preocupación jurídica abarca otras áreas, como el derecho penal. Sus columnas daban amplio eco a la labor de las Cámaras, que, ante los infructuosos intentos de Palacios por regresar al Congreso, adoptaba la forma del comentario, al principio en la pluma del fundador. Una importante sección llevaba por título «Legislación del Trabajo», donde se analizaba con pericia y detalle las normas, incluso decretos, la situación de las cajas, etc., incluyendo además la publicación, en toda su extensión, de las leyes obreras, empezando por la ley $\mathrm{n}^{\circ}$ 9688, de accidentes de trabajo, materia de la que se reproducían asimismo los fallos judiciales. También se seguía la aplicación de las leyes «Palacios» por parte de los tribunales y la administración. Se publicaban incluso proyectos de ley o de ordenanza firmados por otros actores del mundo social, como Alejandro Unsain o Alejandro Ruzo, del Departamento Nacional del Trabajo -el Partido se mostraba abierto a la acción positiva de este organismo, haciendo el elogio de su director Julio B. Lezana, como también del Registro nacional de colocaciones ${ }^{18}-$. De manera general, esto daba cuenta de una mayor interrelación que el viejo ps con las asociaciones sensibles a la cuestión social y al derecho, como el Museo Social Argentino, que venían del tiempo en que Palacios revistaba en sus filas.

Otra prueba de su tropismo legal, era la «sección judicial» de $L A$, con comentarios de fallos, que a veces informaban de los turnos en los juzgados. Sin dejar de estar atento al movimiento extranjero, como era el caso del llamado «buen juez Magnaud", del que se traducen sus sentencias en sucesivos números, ordenadas temáticamente, para el conocimiento del pueblo (lo que justificaba violar los derechos de autor) (HERRERA, 20II) ${ }^{19}$. En otras materias legales, descollaba el apoyo al divorcio. Ya en el ámbito de la agitación, un consultorio jurídico gratuito era propuesto, con gran publicidad, por Palacios y su socio Carlos N. Caminos, detallándose incluso la reseña de las 67 consultas atendidas (cobros de jornales, ciudadanía, divorcio, jubilaciones, exención de servicio militar, etc.).

\footnotetext{
${ }^{18}$ Antonio Rouco Oliva, miembro por entonces del DNT, publicaba a menudo textos sobre el tópico (era hermano de José, que tenía en esos momentos actuación pública en el PS, y que más tarde será uno de los principales actores del PS Independiente).

19 De manera general, las traducciones que ofrecía el periódico eran mayoritariamente de autores franceses, como P. Lafargue o G. Déville, o Zola.
} 
Pero aun cuando se hacía la promoción de la legislación social -como la ley de descanso dominical-, las páginas de $L A$ no olvidaban recordar la importancia de la acción y la unión de las sociedades gremiales. Para el caso, sus hombres insistían que aunque la ley fuera buena, los salarios, la jornada de trabajo, la invalidez, la enfermedad, la ancianidad «son muy malos». La acción gremial capacitaría a la clase obrera para alcanzar sus mejoras, y desde el periódico se exhortaba de manera regular a los trabajadores a organizar sus sindicatos de oficio, o a agremiarse en los ya existentes.

No por nada tampoco sus páginas informaban con mucho detalle sobre el movimiento gremial, empezando por las huelgas, en una sección fija («Acción gremial obrera»), aunque el Partido no parece haber tenido aquí activistas directos más allá de una figura histórica como Vicente Rosáenz, o más tarde el secretario del gremio de bronceros, Carlos Filippa, en todo caso no los reivindicaba como tal ${ }^{20}$. Se apoyaba sin embargo a la FORA, en particular una manifestación en protesta a un fallo condenatorio de sus militantes, donde se aprovechaba para recalcar que la Justicia no estaba en estado de ser otra cosa que un instrumento de dominación. Más tarde Palacios saludará la importancia de la central como actor del Nuevo derecho (HERRERA, 20I8), a la par que defendía «la gran fuerza revolucionaria» de los sindicatos en el contexto de su defensa de la Revolución rusa (PALACios, I92I). Pero el tono no era revolucionario: en notas centrales se alertaba sobre la importancia de los perfiles de los directores de gremio, que tenían una gran responsabilidad, porque en sus manos residía el mejoramiento inmediato de los trabajadores. Retomaban la vieja prédica socialista sobre la responsabilidad en las protestas y en particular de las declaraciones de huelga, aunque, de cara a la situación social, se denunciaba de manera constante el paro masivo y la carestía de vida.

Aquí también el talante reformista era profundizado, buscando retraducir la lucha de clases y la conquista de los poderes públicos en «formulaciones prácticas», en el primer caso como lucha contra los privilegios o dando a los trabajadores la clave de su organización, mientras que como vimos, la lucha política se tornaba acción directa o electoral para obtener la sanción de leyes. Los «trabajadores», de hecho, aparecían interpelados como sujetos por los documentos partidarios luego de los «hombres de buena voluntad» y los «ciudadanos entusiastas».

${ }^{20}$ En la elección de abril de 1918, Rosáenz era presentado por la prensa como el único obrero manual entre los candidatos. En 1920, Filippa integraba la lista a diputados. 
Aunque no se la designaba como tal, la política criolla estaba siempre en su mira, denunciando la dejadez cívica, causante de los males de la República y de esa democracia "perpetuamente incipiente». En un editorial se deploraba que los partidos no se organizasen en torno a programas, como en Europa, sino de hombres. Consideraba que la República tenía necesidad de un «nacionalismo práctico», que liquidase «la falta de ciudadanía de la masa popular». Llamaba para ello a una actuación política realizada con probidad, y con partidos diferenciados. Si, como ya hemos visto, su Programa mínimo proponía la supresión del «fomento artificial de la inmigración», que parecía dirigirse a los trabajadores "golondrina» italianos, propiciaba al mismo tiempo la naturalización de los extranjeros.

En cambio, a diferencia de La Vanguardia ( $L V$ ), las vicisitudes de la Guerra mundial no ocupaban un lugar central en las columnas de $L A$, y si se criticaba la doctrina alemana que rezaba «necesidad es ley», permitiendo la invasión de Bélgica, se aseguraba que era aplicada por todas las potencias beligerantes, incluidas Francia e Inglaterra, para defender sus intereses. No descartaban incluso, siempre en esa vena jurídica ya recalcada, que pudiese servir de antecedente para una Revolución social. Con todo, $L A$ terminaba lamentando la caída de Serbia y denunciando las hordas teutónicas ( $\mathrm{n}^{\circ}$ II). Y la guerra como tal no dejaba nunca de ser denostada como un crimen, en perspectiva alberdiana.

\section{LA DISPUTA CON EL VIEJO PS}

El nacimiento del nuevo partido bajo la bandera de un socialismo nacional iba a llevar rápidamente a la confrontación con su núcleo originario. Las páginas de $L V$ iniciaban la embestida, calificándolos de "amarillos» o aun de "patrioteros», relacionando incluso al grupo con la posición de la SPD mayoritaria, que había apoyado en I9I4 al Gobierno alemán con su voto a los créditos de guerra en el Reichstag. El ps se posicionaba así claramente a su izquierda, considerando que los «Argentinos» desvirtuaban la idea socialista. En efecto, si en un principio se deslegitimaba la empresa aseverando que, a la vista de su Programa, no había justificación para un Partido diferente, pronto les atribuyen una identidad de derechas. La agresividad irá cayendo cuando el PS-A se muestre incapaz de vencerlo en las urnas, aunque se los hacía responsable de la pérdida de su predominio metropolitano a mano de los radicales. 
Desde $L A$ se había predicado la actitud de no responder en ese terreno, pero la profusión de los ataques los obligaba a modificar la táctica, entrando en la invectiva e incluso en el insulto. Por lo pronto, se los tildaba, según los contextos, de "fanáticos», «cuadrados», «dogmáticos», «monopolizadores del partido» o aun de "socialismo elástico», retomando incluso la escala cromática para nombrarlos como «los socialistas del otro color». Más tarde, cuando se produjeron una serie de agresiones físicas en el marco de la campaña electoral de i9ı6, se aludía a ellos como los «moscovitas» en referencia a los «métodos autocráticos» del zarismo. A veces las embestidas se hacían más personales, en particular contra el director de $L V$ en esos momentos, Enrique del Valle Iberlucea, calificado de "furioso internacionalista», burlándose de sus reconocimientos públicos a otros senadores o de la patria, juzgados incompatibles para quien se reivindicaba como revolucionario -lo llamarán después «doctor tira y afloja»-. Incluso la polémica rozaba el mal gusto y la xenofobia, como en un «cuento» de la sección «Tópicos típicos» que ponía en relación a dos dirigentes del viejo partido de origen italiano, Antonio Zaccagnini ("que está criollazo») y Jacinto Oddone («que no puede acriollarse debido a la dureza de sus mandíbulas» ${ }^{21}$, o cuando se dedicaban unos versos infamantes a de Tomaso, firmados por Ramón Gutiérrez. Más tarde llovían críticas para Dickmann, Bravo y Domingo Bessaso, sin hablar de la «familia chefkoviana», un mote que conocerá descendencia.

Tampoco faltaban las comidillas de los conflictos internos, por ejemplo durante los debates del Ix Congreso del ps, para concluir que «las cosas en el partido Socialista han seguido como entonces y aún peor: estatutos anacrónicos, comité de (grandes duques` irresponsables, oligarquía nepótica, censura previa» ${ }^{22}$. Los enfrentamientos no eran sólo de palabra: $L A$ denunciaba las hostilidades en sus actos callejeros, que eran a menudo interrumpidos por afiliados del Ps, llevando incluso a agresiones de hecho, a las que el PS-A amenazaba con poner orden directamente. Al mismo tiempo, se solidarizarán enérgicamente con Justo cuando sufrió el atentado de junio de I916: el suelto denunciaba como carente de sentido el grito del agresor: «soy argentino». Para $L A$, argentinos eran los que trataban de hacer «obra de civilización y de educación de las costumbres», poniendo el ejemplo del

21 «Tópicos típicos», $L A, 15 / 3 / 1916$.

22 "Lo que va de ayer a hoy», $L A, 22 / 3 / 1916$. 
panteón nacional del PS-A (Rivadavia, Alberdi, Sarmiento), y terminaba: «somos argentinos como Justo, pero no como quien trató de asesinarle» ${ }^{23}$.

Ya en la disputa ideológica, José Muzilli, figura juvenil que venía del ala izquierda pero que estaba muy ligado personalmente a Palacios, al tratar de puntualizar en el $\mathrm{n}^{\circ} 2$ las diferencias con el viejo partido en forma de decálogo, apuntaba ante nada que el socialismo no era para los «Argentinos» una religión, rechazando la visión de una doctrina cerrada. Se denostaba así el tutelaje, la jefatura personal y el "predominio de círculos de familia». Más aún, se condenaba el modelo del Jano bifronte, con un programa para adentro y un programa para fuera ${ }^{24}$. El nuevo Partido tendría incluso un mayor carácter internacionalista, ya que no exigía poseer los derechos políticos para ser afiliado ${ }^{25}$. En un artículo firmado por Josué Quesada, se estimaba que la fuerza del ps no era un producto espontáneo de la evolución normal del pueblo sino de sus éxitos electorales. Sus hombres aparecían como disciplinados y sometidos, su programa «largo y confuso ${ }^{26}$. Y por ello temían que el PS-A pueda derrotarlos. Al mismo tiempo se promovía otro modelo de partido, o al menos de discurso, que debía ser claro, de miras cercanas, conciso, rápido. La criticada «indisciplina» de Palacios, su inorganicidad tan vilipendiada se trastocaba en virtud, la libertad: «tanto como está disciplinado el soldado debe estar emancipado el socialista».

De a poco se dibujaba un socialismo que nacía del sentimiento y del corazón y no del dogma. Se insistía en este tópico en otro artículo, donde se aseguraba que la ambición del socialismo era un Estado integrado por demócratas, aspiración que debía ser aplicada a sus propias filas ${ }^{27}$. Se embestía asimismo contra la voluntad de monopolizar todo los puestos por un grupo -calificado nuevamente de «familiar», como ocurriría en los ' 20 con la disidencia del Ps Independiente-, ahogando las voces disidentes, por el control de las instancias partidarias, como el CEN o los congresos. El contra-modelo presentado era el de una organización donde todos los afiliados tenían los mismos derechos, y se apuntaba, entre las diferencias estatutarias, la existencia del Consejo nacional como órgano ejecutivo, que no podía ser

\footnotetext{
23 «El atentado contra Justo», $L A, 30,10 / 6 / 1916$.

24 J. Muzilli, "Nuesto socialismo», $L A, 17 / 11 / 15$.

${ }^{25} \mathrm{~F}$. Ghio, «Internacionalismo 'sui generis'», $L A, 15 / 3 / 1916$.

26 "Nosotros y ellos», LA, 12/01/1916.

27 «Lo que no ven los fanáticos», LA, 19/01/1916.
} 
integrado ni por los parlamentarios ni por el director de la publicación, y donde toda reelección era posible sólo después de un intervalo de 2 años.

$\mathrm{Al}$ mismo tiempo el PS-A no abandonaba las marcas de la tradición, ya sea ilustrando la portada de su periódico con los retratos de los grandes nombres del socialismo europeo, o publicando la letra de «nuestras canciones», que incluían a la Internacional o el Canto de los Trabajadores. Y en orden a su identidad, el nuevo socialismo mantenía como una constante la lucha contra el alcoholismo, o el poder de la religión, defendiendo incluso el ateísmo, y la separación de la Iglesia del Estado, aunque aclarando que se aceptaban afiliados asumían la religión como asunto privado. En el plano cultural, tampoco las páginas del periódico daban cuenta de rupturas: así se informaba de la actividad de las bibliotecas populares, o de la Liga de educación racionalista, y las prácticas de sociabilidad, como los picnic, eran similares. Y como ya lo hemos visto en materia de acción obrera, se seguía promoviendo muy intensamente el cooperativismo. De hecho, el periódico reproducía la carta orgánica de este tipo de institución, anunciando la creación de cooperativas en Campana o Barracas por parte de sus afiliados.

En realidad, los intentos de fundar el ideario del nuevo socialismo daban cuenta de las dificultades por plasmar la identidad nacional en dicha tradición. Por lo pronto, la tónica primera era que el internacionalismo no estaba reñido con la Patria. En el otro extremo, se podía llegar a leer en las columnas del periódico que "propendemos a la formación de una nacionalidad que pondrá orden en el caos que significa el cosmopolitismo, a fin de que, al amparo de la unidad étni$c a$, prosperen las organizaciones gremiales y cooperativas». Es Muzilli, una vez más quien buscaba dilucidar en esos momentos iniciales de la vida partidaria las coordenadas del nuevo partido. Pero esto implicaba también encarar una disputa interna con los recién llegados de la mano de Palacios. En ese sentido, fustigaba a los «lirios rojos», esos profesionales o universitarios que se decían socialistas pero que expresaban más bien una «supervivencia aristocrática», que no debía prosperar en la nueva organización. En otro artículo más sustancioso, Muzilli oponía el nacionalismo aristocrático a la nacionalidad, que se construye en una configuración democrática. Esta resultaba facilitada por las mejoras de las condiciones de trabajo de los inmigrantes, y estaba unida, asimismo, al problema de la tierra y su distribución ${ }^{28}$. También Casaretto recordaba que el internacionalismo

28 «La nacionalidad», LA, 29/12/15. 
era un elemento propio al socialismo, del que no podía prescindirse ${ }^{29}$. Para estos hombres, se trataba menos de una afirmación positiva del nacionalismo, que una redefinición del internacionalismo en términos compatibles con el presente de la nación, puesto que su temporalidad no era el hoy sino el porvenir ${ }^{30}$.

Esto no impedía a otro militante de menor pasado socialista, Domingo Castro Zinny, proclamar en un acto público que, al lado de la pabellón rojo, «que es símbolo de confraternidad entre los pueblos», debía flamear «la bandera argentina, que es el símbolo de una raza fuerte, noble y generosa $\aleph^{31}$. En una carta a la redacción, publicada en el número siguiente, J. Muzilli sostenía por su lado que «nuestro patriotismo no debe fincar en la idolatría estúpida del color de la bandera, sino en la obra de organización económica y cultural del pueblo» ${ }^{32}$. El referente nacional en un proyecto socialista que se quería argentino no se desplegaba sin tensiones.

En una encuesta lanzada por la Revista Argentina de Ciencias Politicas en I9II para clasificar las ideas políticas, los simpatizantes socialistas, que habían respondido en gran número, se pronunciaban masivamente por un «nacionalismo progresivo», opuesto al "nacionalismo histórico». Si se debía relativizar el valor de la opción, puesto que los términos habían sido propuestos por la cédula de la revista, la respuesta indicaba claramente en cuál dirección los socialistas asumían una referencia cada vez más usual en la definición de su ideario político. Al mismo tiempo, nos alerta sobre la conveniencia de distinguir al menos tres elementos distintos en la expresión del componente nacional en la identidad socialista: la elaboración teórico-política de los líderes partidarios, las expresiones concretas de su práctica política (tanto legislativa como partidaria), y los sentimientos, sin duda difusos, de los adherentes y simpatizantes.

\footnotetext{
29 "Socialismo e internacionalismo», $L A, 13 / 5 / 16$.

${ }^{30} \mathrm{M}$. Lapido, «Internacionalismo», $L A, 23 / 2 / 16$.

31 «Actos públicos realizados», $L A, 01 / 3 / 1916$. Como se sabe, la presencia de la bandera argentina había sido una reivindicación «histórica» de Palacios dentro del viejo PS, habiéndose ilustrado una última vez en el Congreso de 1914. Sobre el debate sobre la nacionalidad en el PS previo a la salida de Palacios, ver REYES (2018).

32 «Especie infundada», $L A, 08 / 03 / 16$.
} 
El conflicto con Palacios fue una ocasión para algunos de los principales referentes del ps de puntualizar la relación con el nacionalismo. Mientras Adolfo Dickmann saludaba la salida de estos hombres «que han cubierto sus pequeñas miserias y sus ambiciones con el 'generoso manto nacionalista`, para fingir mejor sus verdaderos propósitos y hacerlos menos reprobables ante los ojos incautos de la masa ignorante» (DICKMANN, I9I6 [1923]: I5), Augusto Bunge cuestionaba que pueda considerarse a Rivadavia como el padre del socialismo, lo que calificaba de ingenuidad (BUNGe, I9I6: I2) ${ }^{33}$. Pero Dickmann prestaba atención en separar las formas morbosas de la verdadera doctrina, que ilustraba con abundantes citas de Justo, de Tomaso, su hermano Enrique y por supuesto Jaurès, sin dejar de celebrar tampoco la «saludable evolución» de Del Valle Iberlucea. También Bunge afirmaba que la nacionalidad no eran las meras tradiciones, sino el presente y el porvenir de un pueblo. En ese sentido, el accionar socialista en su dimensión concreta, «agranda, consolida y dignifica el principio nacional». Para precisar las diferencias, Bunge recordaba el apoyo que los socialistas habían dado en «la guerra europea a sus gobiernos, porque se trataba de defender un conjunto de bienes propios (como las escuelas, la legislación social, cierto bienestar, etc.) que se asociaban a su cultura nacional, un riesgo que no existía entre los trabajadores argentinos» (BUNGE, I9I6: 83).

Pero quizás estemos menos ante una disputa sobre el carácter del nacionalismo que sobre su extensión, o al menos su traducción en la práctica partidaria. El nuevo partido tratará de promoverlo en diferentes grados en pos de diferenciarse del núcleo original. El más abstracto llevaba a la reivindicación de un socialismo concreto, no dogmático, práctico más que teórico, buscando sumarle un estilo político diferente en otros aspectos. Por lo pronto, se promovía una vida interna abierta, sin el ejercicio de un control permanente a base de estatutos, buscando así conjurar la aparición de una clase dirigente fundada en la antigüedad, aunque el fuerte posicionamiento personalista en torno a Palacios no parecía permitirlo,

${ }^{33}$ Celebraba su política avanzada, de la que asumía el carácter práctico. En ese sentido, veía en él un antepasado de la idea de impuesto único, y de alguna manera de «socialismo agrario» (BUNGE, 1916: 29). 
como lo prueba el conflicto interno que estalla a fines de $1916^{34}$. Y la promesa de realizar una obra legislativa que tradujera un reformismo preciso, jurídico, alejado de todo espíritu sectario.

Empero, toda concepción nacional del socialismo suponía, por definición, ampliar la base de la fuerza, que no podía confinarse a los obreros ${ }^{35}$. Afinando cuentas, los periódicos nacionales de 1915 aseveraban que al menos I8.000 votos con los que Palacios había sido electo diputado en I9I2 eran de ciudadanos independientes al socialismo (que no contaría más que con 14.000 votos propios), y así La Razón daba por descontada su reelección en los comicios de abril de I9i6, un cálculo que estaba sin duda en la cabeza de los impulsores del nuevo Partido. Sin embargo, el desmentido importante que el presagio recibirá en tres ocasiones sucesivas, pese a superar en dos de ellas los 30.000 sufragios $^{36}$, era signo que el viejo PS era visto como un proyecto consolidado y orgánico por el electorado de la Ciudad de Buenos Aires. La imposibilidad repetida de hacer elegir a Palacios, y la espectacular merma de sus sufragios a principios de 1920, tendrán una incidencia importante en la corta vida del Ps-A, que apenas obtendrá un concejal en esos ańos (HERRera, 20I8).

De algún modo, la centralidad electoral parecía perturbar la construcción de un socialismo «nacional», en la medida que no se priorizaba en su labor partidaria la elaboración discursiva de ese elemento, y mucho menos se promovía una estrategia política novedosa. Por cierto, no se trataba de un mero oportunismo: el éxito electoral, la posibilidad de alcanzar la representación nacional era necesaria, e incluso imprescindible, para apuntalar la estrategia reformista que alentaba el

\footnotetext{
${ }^{34} \mathrm{~A}$ partir del conflicto entre los órganos nacionales con la federación de la Capital, la más importante del Partido, un congreso convocado por Palacios y el grupo porteño decide la expulsión de los antiguos militantes socialistas que habían fundado el nuevo partido (HERRERA, 2018).

${ }^{35}$ Reaparecían con mayor fuerza los comités independientes que sostenían la plataforma del Partido y, sobre todo, las candidaturas de su líder, lo que no era visto con buenos ojos por los militantes históricos del socialismo, que temían que alimentara el personalismo. No por nada, con mayor fuerza en un primer momento, los órganos del PS-A defienden las tres vías de acción -política, gremial, cooperativa- ya codificadas en la cultura socialista argentina.

36 Palacios no resultará electo en las tres elecciones sucesivas a las que se presenta, en 1916, 1918 y 1920 (HERRERA, 2018).
} 
PS-A. Pero la integración de un componente nacional era de por sí un elemento inestable dentro de la tradición socialista. No por nada, en momentos de la división interna que sufrirá en sus filas del PS-A (HERRERA, 20I8), el ala izquierda buscará relativizar la inscripción "Argentino» hasta hacerla anecdótica, mientras que el sector mayoritario recordaba ahora no sólo la compatibilidad, sino incluso la complementariedad entre nacionalismo e internacionalismo.

En definitiva, la tentativa de fundar una nueva identidad socialista, o al menos una práctica alternativa a la del viejo Ps conocerá dificultades, tanto internas como externas. Entre las primeras, aparecía claramente la imposibilidad para hacer emerger una fuerza alternativa sin una ruptura con la vieja cultura política a la que aquella se encontraba ligada. No bastaba un nuevo programa ${ }^{37}$ si no integraba otro tipo de acción, y por ende de organización ${ }^{38}$. En la larga serie de disidencias que jalonaran el Socialismo, el PS-A fue tal vez el primero que no quiso abandonar una incipiente historia que constituía un capital político, lo que se materializaba con la permanencia del nombre sustantivo, al que sólo se agregaba un aditamento ${ }^{39}$. Tras la sangría de finales de I9I6, el PS-A parecía estar más dispuesto a desligarse de dicha tradición, pero no termina de consolidar una nueva identidad, tal vez porque el partido se volvía cada vez más, tras la salida de militantes históricos, en una maquinaria electoral en torno a Palacios o al nuevo Concejo deliberante, como vía para alcanzar un lugar en la producción legislativa, pero sin lograr constituirse en un proyecto integral.

En definitiva, el PS-A sólo podía representar una intensificación del proyecto reformista, postulando su concepción del socialismo como «obra práctica» y asumiendo más fuertemente su integración en el sistema político argentino, aunque

${ }^{37}$ Y aún en algunas de las polémicas internas del PS-A, que muestra la temprana tensión entre los antiguos afiliados socialistas y los nuevos militantes, se llegaba a afirmar que el programa coincidía "casi punto por punto» con el de la antigua fuerza ( $L A, 23 / 02 / 1916)$.

${ }^{38} \mathrm{Al}$ nacer, el PS-A reivindicaba incluso las mismas formas de propaganda: la tribuna, el periódico y la biblioteca. Era la debilidad del ala izquierda, que rechazaba que el PS-A sea un «gesto», pero al mismo tiempo no lograba proponer una forma o programa alternativo.

${ }^{39}$ Como se hará luego con los adjetivos «Internacional», «Independiente», «Obrero» e incluso, obligado primero por la justicia antes de que se termine asumiendo como tal, «Revolución Nacional». 
en una dimensión -ideológica ante todo- que se revelaba algo abstracta. En ese sentido, se debe profundizar la comparación con la disidencia que io años más tarde encabezará Antonio De Tomaso, incluso en la retórica intrapartidaria -no por nada, los «libertinos» buscarán ganar a Palacios para sus filas, un gesto que se repetirá luego en otras disidencias posteriores-. Asimismo, confrontando el fracaso «Argentino» con el éxito relativo de los Independientes, al menos en vida de su fundador, se ve la dificultad de construir un nuevo Partido desde el llano; el PSI contaban con fogueados dirigentes en todos los ámbitos institucionales, sobre todo en el parlamentario, mientras que la disidencia de Palacios, una vez abandonada la banca, carecía de posiciones e incluso de otros militantes experimentados, con escasas excepciones, como Casaretto o Torcelli, que pronto lo abandonarían ${ }^{40}$.

Las dificultades eran también de orden externo: en momentos en que gobierna una fuerza como el radicalismo yrigoyenista, que hacía del componente nacional una pieza central de su discurso, aquel podía mostrarse menos poderoso, menos punzante para articular una identidad socialista.

La apuesta que implicaba un partido centrado en la figura de Palacios era hacer coincidir las dos narrativas, la socialista y la nacional, en una organización, que al ser novedosa, podía multiplicar los efectos de la antigua identidad, como de hecho ocurrirá al menos con el componente feminista, cuando, en las elecciones de 1920, el PS-A incluya a una mujer en su lista de candidatos, Alcira Riglos de Berón Astrada (HERrera, 20I8). Pero ambas dimensiones podían también esmerilarse, al quedar encerradas en un relato nacional entendido como entramado inter-élites, es decir, como puente con los representantes honestos de las clases dominantes, como ya había quedado de manifiesto en la acción parlamentaria de Palacios (aunque en esta operación tal vez no se diferencie completamente de Justo).

\footnotetext{
${ }^{40}$ Su temperamento individualista, por otro lado, no era el de un constructor de partido. Hacia fines de Ios años 1910, en la medida que su figura se consolida cada vez más en otros ámbitos, en particular el universitario -tanto en su fase política tras la Reforma universitaria que lo coloca en un rol dirigente, como en la académica, con la consolidación de su prestigio en las cátedras de Buenos Aires y La Plata- su interés por la política partidaria parece decaer aún más.
} 
Por cierto, la atracción de lo nacional para refundar el proyecto socialista no era una inquietud propiamente argentina sino el producto de la crisis del socialismo europeo que se materializaba en 1914. No por nada, al final de la Guerra nacían variados intentos por redefinir el ideario socialista a partir de lo nacional, como lo expresaba en Francia, por un corto tiempo, la figura de un Edmond Laskine ${ }^{41}$. Hemos observado ya que el enfrentamiento entre socialistas se asociaba a las propias vicisitudes que estaba viviendo el socialismo internacional, con la tentación de ubicar al PS-A en una dinámica nacionalista cercana al ala mayoritaria de la SPD. Los "Argentinos», por el contrario, veían en la crisis del socialismo de la II Internacional y su incapacidad de concretar sus postulados internacionalistas, una prueba de la importancia de la nación.

En la nueva doctrina política que se buscaba fundar, la patria aparecía como un elemento intermedio, entre el "pueblo», categoría que termina siendo preferida a la de "proletariado», y la «humanidad» como destino final, como si esta última fuera demasiado abstracta para ser encarnada concretamente. Al mismo tiempo, la nacionalidad, omnipresente en el discurso, era algo en devenir, en aras de ser forjado por la acción de argentinos y extranjeros. En todo caso, la patria podía aparecer como una escala política en un camino más vasto que no se detenía en la liberación nacional. De manera embrionaria, aparece ya la nación no como origen, sino como "proyección emancipatoria»" ${ }^{42}$. Pero el propio Palacios parecía moderar esta visión al final de la Guerra, tal vez ante las promesas de las nuevas organizaciones internacionales para la protección del trabajo, así como la consolidación de la Revolución rusa.

${ }^{41}$ Ya Manuel Ugarte había buscado definir con mayor precisión la relación entre socialismo y patria (entendida como «el derecho que tienen todos los núcleos sociales de vivir a su manera y a disponer de su suerte») en un artículo de 1908 publicado en $L V$ tras el Congreso de Stuttgart (UGARTE, 1914). Al mismo tiempo proyectaba su visión de América latina en un «continentalismo» iberoamericano. Una polémica en ese sentido con E. Dickmann, unos años después, le ganaba el apoyo público de Alfredo Torcelli, pero puntuada por una cuestión de honor con Palacios, termina con su salida del PS. En el Manifiesto donde explicaba su partida subrayaba su calidad de argentino, que antecede su interés por la humanidad. Basaba su «moderado» socialismo en la colaboración y no la lucha de clases, negándose a sostener la abolición de la propiedad privada y llamando al respeto del Ejército y la religión. La Gran Guerra parecía alejarlo aún más de la perspectiva socialista. De hecho, M. Casaretto polemizará con Ugarte desde las columnas de $L A$, sosteniendo que la guerra mundial no había hecho fracasar al socialismo. Con todo reconocía sin dificultad que la nación no era sólo el punto de partida sino también de apoyo para el socialismo, reivindicando una vez más a Jaurès 42 Para retomar una fórmula reciente de OMAR ACHA (2017: 135). 
El fracaso del PS-A en recrear una nueva identidad socialista no fue distinto del que conocieron las otras experiencias partidarias que, como el PSI o el PSO, hicieron del elemento "nacional» un componente de un proyecto más amplio, como si aquel fracaso hubiera calado en la izquierda argentina más allá de las épocas. Al menos marcará a su promotor, quien, tras mostrar cierta crítica de los partidos políticos en nombre del carácter revolucionario de las organizaciones obreras, que se traducía por una defensa tanto de la Fora como institución como de la Revolución Rusa, en clara distancia, en ambos casos, con la retórica socialdemócrata en esos primeros años de la década de 1920 , se retiró durante casi una década de la actividad partidaria, aunque permaneciendo activo de manera inversamente proporcional en las organizaciones culturales y universitarias. A su regreso al viejo Ps, Palacios ya no buscó proyectar una identidad concurrente, contentándose con cultivar su propia singularidad dentro del universo partidario. 


\section{Referencias bibliográficas}

AA. W. (1915): El Diputado Palacios. Su separación del Partido Socialista, Buenos Aires, Rosso. ACHA, OMAR (2017): Cambiar de ideas. Cuatro tentativas sobre Oscar Terán, Buenos Aires, Prometeo.

ARICÓ, JOSÉ (1998): La hipótesis de Justo, Buenos Aires, Planeta.

BERNSTEIN, SERGE (1998): «La culture politique», en: J.P. Rioux, J. F. Sirinelli, (eds.), Pour une histoire culturelle, París, Seuil.

BERNSTEIN, SERGE (1999): «Nature et fonctions des cultures politiques», in Id. (ed.), Les cultures politiques en France, París, Seuil.

BUNGE, AUGUSTO (1916): El ideal argentino y el socialismo, Buenos Aires, La Vanguardia.

CAMARERO, HERNÁN Y HERRERA, CARLOS MIGUEL (2005):

El Partido Socialista en Argentina. Sociedad, política e ideas a través de un sig/o, Buenos Aires, Prometeo.

DICKMANN, ADOLFO (1933 [1916]): Nacionalismo y Socialismo, Buenos Aires, Porter.

DICKMANN, ENRIQUE (1917): Democracia y Socialismo, Buenos Aires, Ponzinibbio.

GARCÍA COSTA, VÍCTOR (1998): Alfredo Palacios, entre el clavel y la espada. Una biografía, Buenos Aires, Planeta.

HERRERA, CARLOS MIGUEL (2011): «Entre équité et socialisme: le juge et la question sociale dans le débat politico-doctrinal français du début du XX siècle», en: Quaderni Fiorentini per la storia del pensiero giuridico moderno, $n^{\circ}$ 40, p. 331-395.
HERRERA, CARLOS MIGUEL (2015): «Socialismo, democracia, derecho: de Jean Jaurès a Juan B. Justo», en: A. Lazzeretti y F. Suárez (eds.), Socialismo \& democracia, Mar del Plata, EUDEM, p. 193-219. HERRERA, CARLOS MIGUEL (2016): ¿Adios al proletariado? El Partido Socialista bajo el peronismo, Buenos Aires, Imago Mundi.

HERRERA, CARLOS MIGUEL (2018): «El frustrado accionar de un partido socialista nacional en Argentina (1916-1922)», en: Archivos de historia del movimiento obrero y la izquierda, $\mathrm{n}^{\circ} 13, \mathrm{p} .121-141$. JUSTO, JUAN B. (1909): Teoría y práctica de la historia, Buenos Aires, Lotitto.

JUSTO, JUAN B. (1923): «Por qué no me gusta escribir para una hoja que se dice israelita», en: Nosotros. PALACIOS, ALFREDO (1916): En defensa de los trabajadores - El Partido Socialista Argentino, Buenos Aires, Prometeo.

PALACIOS, ALFREDO (1921): La Revolución Rusa, Buenos Aires, Agencia Sudamericana de Libros. REYES, FRANCISCO (2018): «EI Jano socialista. Juan B. Justo y el lugar de los símbolos en la política moderna», en: Estudios Sociales, no 55.

SIRINELLI, JEAN FRANÇOIS (1998): «Eloge de la complexité», en : Jean Pierre Rioux y Jean François Sirinelli (eds.), Pour une histoire culturelle, París, Seuil. TORRE, JUAN CARLOS (1976): «La primera victoria electoral», en: Todo es Historia.

UGARTE, MANUEL (1914): Manuel Ugarte y el Partido Socialista, Buenos Aires, Unión Editorial Hispano-América. 